\title{
Iterative Solutions for the Differential Equation with $p$-Laplacian on Infinite Interval
}

\author{
Guoying Zhao ${ }^{1}$ and Ying Wang $\mathbb{D}^{2}$ \\ ${ }^{1}$ School of Logistics, Linyi University, Linyi, 276000 Shandong, China \\ ${ }^{2}$ School of Mathematics and Statistics, Linyi University, Linyi, 276000 Shandong, China
}

Correspondence should be addressed to Ying Wang; lywy1981@163.com

Received 25 April 2021; Accepted 12 June 2021; Published 25 June 2021

Academic Editor: Fanglei Wang

Copyright (c) 2021 Guoying Zhao and Ying Wang. This is an open access article distributed under the Creative Commons Attribution License, which permits unrestricted use, distribution, and reproduction in any medium, provided the original work is properly cited.

This paper systematically investigates a class of fourth-order differential equation with $p$-Laplacian on infinite interval in Banach space. By means of the monotone iterative technique, we establish not only the existence of positive solutions but also iterative schemes under the suitable conditions. At last, we give an example to demonstrate the application of the main result.

\section{Introduction}

The partial differential equation with the $p$-Laplacian operator

$$
-\operatorname{div}\left(\left|\nabla_{u}(x)\right|^{p-2} \nabla u(x)\right)+b(x) \varphi(u(x))=0,
$$

which is often used to describe, for example, diffusion process [1], with a spatial symmetric potential $b$, can be reduced to $\left(r(t) \varphi_{p}\left(y^{\prime}(t)\right)\right)^{\prime}+c(t) \varphi_{p}(y(t))=0$, where $\varphi_{p}(x)=|x|^{p-2} x, p$ $>1$. This fact leads us to study the following $p$-Laplacian boundary value problem (BVP):

$$
\left\{\begin{array}{l}
\left(\varphi_{p}\left(u^{\prime \prime}(t)\right)\right)^{\prime \prime}-k^{2} \varphi_{p}\left(u^{\prime \prime}(t)\right)=a(t) f(t, u(t)), \in(0,+\infty), \\
u(0)=\int_{0}^{\infty} g(t) u(t) d t, \lim _{t \longrightarrow+\infty} u^{\prime}(t)=\xi, u^{\prime \prime}(0)=\lim _{t \rightarrow+\infty} u^{\prime \prime}(t)=0,
\end{array}\right.
$$

where $\varphi_{p}(x)=|x|^{p-2} x, p>1, \varphi_{q}=\varphi_{p}^{-1},(1 / p)+(1 / q)=1, k>0$ ,$\xi \geq 0$ are real constants, $g:[0,+\infty) \longrightarrow[0,+\infty)$ is a Lebesgue integrable function with $\int_{0}^{\infty} g(t) d t<1, \int_{0}^{\infty} \operatorname{tg}(t) d t<+\infty$ , $a:(0,+\infty) \longrightarrow[0,+\infty)$ is continuous and may be singular at $t=0$, and $f:[0,+\infty) \times[0,+\infty) \longrightarrow[0,+\infty)$ is a continuous function.
The $p$-Laplacian equation arises quite naturally in the modeling of different physical and natural phenomena. For instance, in fluid mechanics, the shear stress $\vec{\tau}$ and the velocity gradient $\nabla_{p} u$ of certain fluids obey a relation of the form $\vec{\tau}=a(x) \nabla_{p} u$, where $\nabla_{p} u=\left|\nabla_{u}\right|^{p-2} \nabla u$. Here, the real number $p>1$ and $p=2$ (respectively, $p<2, p>2$ ) designate a Newtonian (respectively, pseudoplastic, dilatant) fluid. Given $a$ is a constant, the resulting equations of motion then involve $\operatorname{div}\left(a \nabla_{p} u\right)$, which reduces to $a \Delta_{p} u=a \operatorname{div} \nabla_{p} u$. Over the last couple of decades, many important results including integral and fractional equations with $p$-Laplacian on certain boundary value conditions had been obtained. We refer the reader to [2-17] and the references cited therein. Liang and Zhang [18] considered the $m$-point BVP with a p-Laplacian operator

$$
\left\{\begin{array}{l}
\left(\varphi_{p}\left(u^{\prime}(t)\right)\right)^{\prime}+h(t) f(u(t))=0, t \in(0,+\infty), \\
u(0)=\sum_{i=1}^{m-2} \alpha_{i} u\left(\xi_{i}\right), \lim _{t \rightarrow+\infty} u^{\prime}(t)=0
\end{array}\right.
$$

where $\xi_{i} \in(0,+\infty)$, with $0<\xi_{1}<\xi_{2}<\cdots<\xi_{m-2}<+\infty$, and $\alpha_{i}$ satisfies $\alpha_{i} \in[0,+\infty), \quad 0 \leq \sum_{i=1}^{m-2} \alpha_{i}<1, h:[0,+\infty) \longrightarrow[0$, $+\infty)$ and has countably many singularities in $[1,+\infty), f:[$ 
$0,+\infty) \longrightarrow[0,+\infty)$ is a continuous function. The existence of positive solutions is obtained by applying the fixed-point theorem of Leggett-Williams.

Using the fixed point index theory, Xu and Yang in [19] researched the existence of positive solutions for the fourth order $p$-Laplacian BVP

$$
\left\{\begin{array}{l}
\left(\left|u^{\prime \prime}\right|^{p-1} u^{\prime \prime}\right)^{\prime \prime}=f(t, u), t \in(0,1), \\
u^{(2 i)}(0)=u^{(2 i)}(1)=0, i=0,1,
\end{array}\right.
$$

where $p>0, f:[0,1] \times[0,+\infty) \longrightarrow[0,+\infty)$ is a continuous function.

The motivation for the present work stems from both practical and theoretical aspects. In fact, many mathematical problems in science and engineering are set in unbounded domains, such as unsteady flow of gas through a semiinfinite porous media, the theory of drain flows, plasma physics, in determining the electrical potential in an isolated neutral atom. In all these applications, it is frequent that only positive solutions are useful. In this paper, we study the differential equation with $p$-Laplacian operator as BVP (2); when $p=2$, BVP (2) becomes the ordinary fourth-order differential equation. The results for the existence of the maximal and minimal solutions to the BVP (2) are established. In addition, we establish iterative schemes for approximating the solutions, which start from the known simple linear functions. However, to the best knowledge of the authors, there are few works in the literature dealing with the existence of positive solutions to boundary value problems of differential equation on infinite intervals with $p$-Laplacian operator by using iterative technique up to now. The goal of the present paper is to fill the gap in this area, so it is interesting and important to study the existence of positive solutions for BVP (2).

\section{Preliminaries and Lemmas}

The basic space used in this paper is $E$, where $E$ is denoted by

$$
E=\left\{u \in C[0,+\infty): \sup _{t \in[0,+\infty)} \frac{|u(t)|}{1+t}<+\infty\right\}
$$

Then, $E$ is a Banach space equipped with the norm $\|u\|$ $=\sup _{t \in[0,+\infty)}(|u(t)| /(1+t))$. Define a cone $K$ in the Banach space $E$ by $K=\{u \in E: u(t) \geq 0, t \in[0,+\infty)\}$.

Lemma 1 (see [20]). Let $y \in C[0,+\infty) \cap L^{1}(0,+\infty)$, then $x$ is a solution of

$$
\left\{\begin{array}{l}
x^{\prime \prime}(t)-k^{2} x(t)+y(t)=0, t \in(0,+\infty), \\
x(0)=\lim _{t \longrightarrow+\infty} x(t)=0,
\end{array}\right.
$$

if and only if $x$ is a solution of

$$
x(t)=\int_{0}^{\infty} G(t, s) y(s) d s,
$$

where

$$
G(t, s)=\frac{1}{2 k}\left\{\begin{array}{l}
e^{-k s}\left(e^{k t}-e^{-k t}\right), 0 \leq t \leq s<+\infty, \\
e^{-k t}\left(e^{k s}-e^{-k s}\right), 0 \leq s \leq t<+\infty .
\end{array}\right.
$$

Lemma 2. Green's function $G(t, s)$ satisfies

(1) $G(t, s) \geq 0$, for any $t, s \in[0,+\infty)$

(2) $G(t, s) \leq G(s, s) \leq 1 / 2 k$, for any $t, s \in[0,+\infty)$

In what follows, we list some conditions for convenience.

$\left(H_{0}\right) g:[0,+\infty) \longrightarrow[0,+\infty)$ is a Lebesgue integrable function with $\int_{0}^{\infty} g(t) d t<1, \int_{0}^{\infty} \operatorname{tg}(t) d t<+\infty$.

$\left(H_{1}\right) f:[0,+\infty) \times[0,+\infty) \longrightarrow[0,+\infty)$ is a continuous function, $f(t, 0) \equiv 0$ on $[0,+\infty)$ and $f(t,(1+t) x)$ is bounded, for $t \in[0,+\infty), x \in D, D \subset[0,+\infty)$ is a closed subinterval.

$\left(H_{2}\right) a:(0,+\infty) \longrightarrow[0,+\infty)$ is continuous, $a(t) \equiv 0$ on $[0,+\infty)$ and

$$
\begin{aligned}
0 & <\int_{0}^{\infty} G(s, s) a(s) d s \\
& <+\infty, \int_{0}^{\infty} s \varphi_{q}\left(\int_{0}^{\infty} G(s, \varsigma) a(\varsigma) d \varsigma\right) d s<+\infty .
\end{aligned}
$$

By routine discussion, Lemma 3 is valid.

Lemma 3. Assume that $\left(H_{0}\right)$ holds and $\varphi_{q}(x) \in C[0,+\infty) \cap$ $L^{1}(0,+\infty)$; then, $u$ is a solution of

$$
\left\{\begin{array}{l}
u^{\prime \prime}(t)+\varphi_{q}(x(t))=0, t \in(0,+\infty), \\
u(0)=\int_{0}^{\infty} g(t) u(t) d t, \lim _{t \rightarrow+\infty} u^{\prime}(t)=\xi,
\end{array}\right.
$$

if and only if $u$ is a solution of

$$
\begin{aligned}
u(t)= & \frac{1}{1-\int_{0}^{\infty} g(t) d t}\left(\xi \int_{0}^{\infty} \operatorname{tg}(t) d t\right. \\
& \left.+\int_{0}^{\infty} g(t) \int_{0}^{t} \int_{s}^{\infty} \varphi_{q}(x(\tau)) d \tau d s d t\right) \\
& +\int_{0}^{t} \int_{s}^{\infty} \varphi_{q}(x(\tau)) d \tau d s+\xi t
\end{aligned}
$$

Let $x(t)=-\varphi_{p}\left(u^{\prime \prime}(t)\right)$, then BVP (2) is divided into the following two parts: 


$$
\begin{aligned}
& \left\{\begin{array}{l}
x^{\prime \prime}(t)-k^{2} x(t)+a(t) f(t, u(t))=0, t \in(0,+\infty), \\
x(0)=\lim _{t \longrightarrow+\infty} x(t)=0,
\end{array}\right. \\
& \left\{\begin{array}{l}
u^{\prime \prime}(t)+\varphi_{q}(x(t))=0, t \in(0,+\infty), \\
u(0)=\int_{0}^{\infty} g(t) u(t) d t, \lim _{t \rightarrow+\infty} u^{\prime}(t)=\xi .
\end{array}\right.
\end{aligned}
$$

From Lemmas 1 and 3, under the above assumptions $\left(H_{0}\right)-\left(H_{2}\right)$, denote the operator $A: K \longrightarrow E$ as follows:

$$
\begin{aligned}
A u(t)= & \frac{1}{1-\int_{0}^{\infty} g(t) d t}\left(\xi \int_{0}^{\infty} t g(t) d t+\int_{0}^{\infty} g(t) \int_{0}^{t} \int_{s}^{\infty} \varphi_{q}\right. \\
& \left.\cdot\left(\int_{0}^{\infty} G(\tau, \varsigma) a(\varsigma) f(\varsigma, u(\varsigma)) d \varsigma\right) d \tau d s d t\right) \\
& +\int_{0}^{t} \int_{s}^{\infty} \varphi_{q}\left(\int_{0}^{\infty} G(\tau, \varsigma) a(\varsigma) f(\varsigma, u(\varsigma)) d \varsigma\right) d \tau d s \\
& +\xi t, t \in[0,+\infty) .
\end{aligned}
$$

Now, we claim that $A u$ is well defined for $u \in K$. In fact, for any $u \in K$, there exists $r>0$, such that $\mid(u(t)) /(1$ $+t) \mid \leq r, t \in[0,+\infty)$. From $\left(H_{1}\right)$ and the definition of $\|\cdot\|$, we have

$$
S_{f}^{r}:=\sup \{f(t,(1+t) x):(t, x) \in[0,+\infty) \times[0, r]\}<+\infty .
$$

Thus, by $\left(H_{1}\right)\left(H_{2}\right)$, we know

$$
\begin{aligned}
& \int_{0}^{t} \int_{s}^{\infty} \varphi_{q}\left(\int_{0}^{\infty} G(\tau, \varsigma) a(\varsigma) f(\varsigma, u(\varsigma)) d \varsigma\right) d \tau d s \\
& \quad \leq \varphi_{q}\left(S_{f}^{r}\right) \int_{0}^{\infty} s \varphi_{q}\left(\int_{0}^{\infty} G(s, \varsigma) a(\varsigma) d \varsigma\right) d s<+\infty
\end{aligned}
$$

Since

$$
\int_{0}^{\infty} g(t) d t<1, \int_{0}^{\infty} \operatorname{tg}(t) d t<+\infty
$$
that

Together with (15), for any $t \in[0,+\infty)$, we can see

$$
\begin{aligned}
\frac{|A u(t)|}{1+t} \leq & \frac{1}{1-\int_{0}^{\infty} g(t) d t}\left(\xi \int_{0}^{\infty} \operatorname{tg}(t) d t+\int_{0}^{\infty} g(t) \int_{0}^{t} \int_{s}^{\infty} \varphi_{q}\right. \\
& \left.\cdot\left(\int_{0}^{\infty} G(\tau, \varsigma) a(\varsigma) f(\varsigma, u(\varsigma)) d \varsigma\right) d \tau d s d t\right) \\
& +\int_{0}^{t} \int_{\varsigma}^{\infty} \varphi_{q}\left(\int_{0}^{\infty} G(\tau, \varsigma) a(\varsigma) f(\varsigma, u(\varsigma)) d \varsigma\right) d \tau d s+\xi
\end{aligned}
$$

$$
\begin{aligned}
\leq & \frac{1}{1-\int_{0}^{\infty} g(t) d t}\left(\xi \int_{0}^{\infty} \operatorname{tg}(t) d t+\varphi_{q}\left(S_{f}^{r}\right) \int_{0}^{\infty} s \varphi_{q}\right. \\
& \left.\cdot\left(\int_{0}^{\infty} G(s, \varsigma) a(\varsigma) d \varsigma\right) d s \int_{0}^{\infty} g(t) d t\right) \\
& +\varphi_{q}\left(S_{f}^{r}\right) \int_{0}^{\infty} s \varphi_{q}\left(\int_{0}^{\infty} G(s, \varsigma) a(\varsigma) d \varsigma\right) d s+\xi
\end{aligned}
$$

So, by (15) and (17), we obtain

$$
\sup _{t \in[0,+\infty)} \frac{|A u(t)|}{1+t}<+\infty .
$$
have

On the other hand, for any $t_{1}, t_{2} \in[0,+\infty)$, by (13), we

$$
\begin{aligned}
& \left|A u\left(t_{1}\right)-A u\left(t_{2}\right)\right| \\
& \leq\left|\int_{t_{1}}^{t_{2}} \int_{s}^{\infty} \varphi_{q}\left(\int_{0}^{\infty} G(\tau, \varsigma) a(\varsigma) f(\varsigma, u(\varsigma)) d \varsigma\right) d \tau d s\right| \\
& \quad+\xi\left|t_{1}-t_{2}\right| \longrightarrow 0, \text { as } t_{1} \longrightarrow t_{2} .
\end{aligned}
$$

Therefore, $A u \in C[0,+\infty)$, for any $u \in K$. Hence, $A$ $: K \longrightarrow E$ is well defined. Obviously, $u$ is a positive solution of BVP (2) if and only if $u$ is a fixed point of $A$ in $K$.

The Arzela-Ascoli theorem fails to work in the Banach space $E$ due to the fact that the infinite interval $[0,+\infty)$ is noncompact. The following compactness criterion will help us to resolve this problem.

Lemma 4 (see $[21,22])$. Let $E$ be defined as (5) and $M$ be any bounded subset of $E$. Then, $M$ is relatively compact in $E$ if $\{x(t) /(1+t): x \in M\}$ is equicontinuous on any finite subinterval of $[0,+\infty)$, and for any given $\varepsilon>0$, there exists $N>0$, such that $\left|\left(x\left(t_{1}\right) /\left(1+t_{1}\right)\right)-\left(x\left(t_{2}\right) /\left(1+t_{2}\right)\right)\right|<\varepsilon$ uniformly with respect to $x \in M$, as $t_{1}, t_{2}>N$.

\section{Main Results}

Lemma 5. Assume that $\left(\mathrm{H}_{0}\right)-\left(\mathrm{H}_{2}\right)$ hold. Then, $A: K \longrightarrow K$ is completely continuous.

Proof. It is clear that $A u(t) \geq 0$ for any $u \in K, t \in[0,+\infty)$. Thus, $A(K) \subseteq K$. Now, we prove that $A$ is continuous and compact, respectively. Let $M \subset K$ be a bounded subset. Then, there exists $R>0$, such that $\|x\|<R$, for any $x \in M$. So, for any $x \in M$, we have 


$$
\begin{aligned}
\|A u\|= & \sup _{t \in[0,+\infty)} \frac{1}{1+t} \mid \frac{1}{1-\int_{0}^{\infty} g(t) d t}\left(\xi \int_{0}^{\infty} t g(t) d t+\int_{0}^{\infty} g(t)\right. \\
& \left.\cdot \int_{0}^{t} \int_{s}^{\infty} \varphi_{q}\left(\int_{0}^{\infty} G(\tau, \varsigma) a(\varsigma) f(\varsigma, u(\varsigma)) d \varsigma\right) d \tau d s d t\right) \\
& +\int_{0}^{t} \int_{s}^{\infty} \varphi_{q}\left(\int_{0}^{\infty} G(\tau, \varsigma) a(\varsigma) f(\varsigma, u(\varsigma)) d \varsigma\right) d \tau d s+\xi t \mid \\
\leq & \frac{1}{1-\int_{0}^{\infty} g(t) d t}\left(\xi \int_{0}^{\infty} t g(t) d t+\varphi_{q}\left(S_{f}^{R}\right) \int_{0}^{\infty} s \varphi_{q}\right. \\
& \left.\cdot\left(\int_{0}^{\infty} G(s, \varsigma) a(\varsigma) d \varsigma\right) d s \int_{0}^{\infty} g(t) d t\right) \\
& +\varphi_{q}\left(s_{f}^{R}\right) \int_{0}^{\infty} s \varphi_{q}\left(\int_{0}^{\infty} G(s, \varsigma) a(\varsigma) d \varsigma\right) d s+\xi<+\infty
\end{aligned}
$$

where

$$
S_{f}^{R}:=\sup \{f(t,(1+t) x):(t, x) \in[0,+\infty) \times[0, R]\}<+\infty .
$$

So, $A M$ is bounded in $E$. Moreover, given $T \in(0,+\infty)$, for any $x \in M$ and $t_{1}, t_{2} \in[0, T]$, without loss of generality, we may assume that $t_{1}<t_{2}$. In fact,

$$
\begin{aligned}
& \left|\frac{A u\left(t_{1}\right)}{1+t_{1}}-\frac{A u\left(t_{2}\right)}{1+t_{2}}\right| \\
& \leq\left|\frac{1}{1+t_{1}}-\frac{1}{1+t_{2}}\right| \frac{1}{1-\int_{0}^{\infty} g(t) d t}\left(\xi \int_{0}^{\infty} t g(t) d t+\int_{0}^{\infty} g(t)\right. \\
& \left.\quad \cdot \int_{0}^{t} \int_{s}^{\infty} \varphi_{q}\left(\int_{0}^{\infty} G(\tau, \varsigma) a(\varsigma) f(\varsigma, u(\varsigma)) d \varsigma\right) d \tau d s d t\right) \\
& \quad+\mid \frac{1}{1+t_{1}} \int_{0}^{t_{1}} \int_{s}^{\infty} \varphi_{q}\left(\int_{0}^{\infty} G(\tau, \varsigma) a(\varsigma) f(\varsigma, u(\varsigma)) d \varsigma\right) d \tau d s \\
& \quad-\frac{1}{1+t_{2}} \int_{0}^{t_{2}} \int_{s}^{\infty} \varphi_{q}\left(\int_{0}^{\infty} G(\tau, \varsigma) a(\varsigma) f(\varsigma, u(\varsigma)) d \varsigma\right) d \tau d s \mid \\
& \quad+\xi\left|\frac{t_{1}}{1+t_{1}}-\frac{t_{2}}{1+t_{2}}\right| \leq\left|\frac{1}{1+t_{1}}-\frac{1}{1+t_{2}}\right| \frac{1}{1-\int_{0}^{\infty} g(t) d t} \\
& \quad \cdot\left(\xi \int_{0}^{\infty} t g(t) d t+\varphi_{q}\left(S_{f}^{R}\right) \int_{0}^{\infty} g(t) d t \int_{0}^{\infty} s \varphi_{q}\right. \\
& \left.\quad \cdot\left(\int_{0}^{\infty} G(s, \varsigma) a(\varsigma) d \varsigma\right) d s\right)+\left|\frac{1}{1+t_{1}}-\frac{1}{1+t_{2}}\right| \varphi_{q}\left(s_{f}^{R}\right) \\
& \quad \cdot \int_{0}^{t_{1}} \int_{s}^{\infty} \varphi_{q}\left(\int_{0}^{\infty} G(\tau, \varsigma) a(\varsigma) d \varsigma\right) d \tau d s \\
& \quad+\varphi_{q}\left(s_{f}^{R}\right) \int_{t_{1}}^{t_{2}} \int_{s}^{\infty} \varphi_{q}\left(\int_{0}^{\infty} G(\tau, \varsigma) a(\varsigma) d \varsigma\right) d \tau d s \\
& +\xi\left|\frac{t_{1}}{1+t_{1}}-\frac{t_{2}}{1+t_{2}}\right| .
\end{aligned}
$$

So, for any $\varepsilon>0$, there exists $\delta>0$, such that for any $t_{1}$, $t_{2} \in[0, T]$ with $\left|t_{1}-t_{2}\right|<\delta$, and for any $x \in M$, we have

$$
\left|\frac{(A u)\left(t_{1}\right)}{1+t_{1}}-\frac{(A u)\left(t_{2}\right)}{1+t_{2}}<\right| \delta .
$$

Hence, $\{(A u)(t) /(1+t): u \in M\}$ is equicontinuous on $[0, T]$. Since $T>0$ is arbitrary, $\{(A u)(t) /(1+t): u \in M\}$ is locally equicontinuous on $[0,+\infty)$.

Next, we prove that we show that $A: K \longrightarrow K$ is equiconvergent at $+\infty$. For any $u \in M$, we have

$$
\begin{aligned}
\lim _{t \longrightarrow+\infty}\left|\frac{A u(t)}{1+t}\right| & \\
= & \lim _{t \longrightarrow+\infty} \frac{1}{1+t} \mid \frac{1}{1-\int_{0}^{\infty} g(t) d t}\left(\xi \int_{0}^{\infty} t g(t) d t+\int_{0}^{\infty} g(t)\right. \\
& \left.\quad \int_{0}^{t} \int_{s}^{\infty} \varphi_{q}\left(\int_{0}^{\infty} G(\tau, \varsigma) a(\varsigma) f(\varsigma, u(\varsigma)) d \varsigma\right) d \tau d s d t\right) \\
& +\int_{0}^{t} \int_{s}^{\infty} \varphi_{q}\left(\int_{0}^{\infty} G(\tau, \varsigma) a(\varsigma) f(\varsigma, u(\varsigma)) d \varsigma\right) d \tau d s+\xi t \mid \\
= & \lim _{t \rightarrow+\infty} \int_{t}^{\infty} \varphi_{q}\left(\int_{0}^{\infty} G(\tau, \varsigma) a(\varsigma) f(\varsigma, u(\varsigma)) d \varsigma\right) d \tau+\xi=\xi .
\end{aligned}
$$

So, for any $u \in M$, we have

$$
\begin{aligned}
\left|\frac{A u(t)}{1+t}-\xi\right| & \mid \frac{1}{1+t}\left(\frac { 1 } { 1 - \int _ { 0 } ^ { \infty } g ( t ) d t } \left(\xi \int_{0}^{\infty} t g(t) d t+\int_{0}^{\infty} g(t)\right.\right. \\
& \left.\cdot \int_{0}^{t} \int_{s}^{\infty} \varphi_{q}\left(\int_{0}^{\infty} G(\tau, \varsigma) a(\varsigma) f(\varsigma, u(\varsigma)) d \varsigma\right) d \tau d s d t\right) \\
& \left.+\int_{0}^{t} \int_{s}^{\infty} \varphi_{q}\left(\int_{0}^{\infty} G(\tau, \varsigma) a(\varsigma) f(\varsigma, u(\varsigma)) d \varsigma\right) d \tau d s+\xi t\right)-\xi \mid \\
\leq & \frac{1}{1+t}\left(\frac { 1 } { 1 - \int _ { 0 } ^ { \infty } g ( t ) d t } \left(\xi \int_{0}^{\infty} t g(t) d t+\varphi_{q}\left(s_{f}^{R}\right)\right.\right. \\
& \left.\cdot \int_{0}^{\infty} s \varphi_{q}\left(\int_{0}^{\infty} G(s, \varsigma) a(\varsigma) d \varsigma\right) d s \int_{0}^{\infty} g(t) d t\right) \\
& \left.+\varphi_{q}\left(s_{f}^{R}\right) \int_{0}^{\infty} s \varphi_{q}\left(\int_{0}^{\infty} G(s, \varsigma) a(\varsigma) d \varsigma\right) d s\right) \\
& -\frac{\xi}{1+t} \mid \longrightarrow 0, t \longrightarrow+\infty .
\end{aligned}
$$

Thus, for any $\varepsilon>0$, there exists $N>0$, for any $t>N$ and for any $x \in M$, such that

$$
\left|\frac{A u(t)}{1+t}-\xi\right|<\frac{\varepsilon}{2} .
$$



have

Consequently, for any $t_{1}, t_{2}>N$ and for any $x \in M$, we

$$
\begin{aligned}
\left|\frac{A u\left(t_{1}\right)}{1+t_{1}}-\xi\right| & <\frac{\varepsilon}{2}, \\
\left|\frac{A u\left(t_{2}\right)}{1+t_{2}}-\xi\right| & <\frac{\varepsilon}{2}, \\
t_{1}, t_{2} & >N .
\end{aligned}
$$

Therefore, for any $t_{1}, t_{2}>N$ and for any $x \in M$, we have

$$
\begin{aligned}
\left|\frac{A u\left(t_{1}\right)}{1+t_{1}}-\frac{A u\left(t_{2}\right)}{1+t_{2}}\right| & =\left|\left(\frac{A u\left(t_{1}\right)}{1+t_{1}}-\xi\right)-\left(\frac{A u\left(t_{2}\right)}{1+t_{2}}-\xi\right)\right| \\
& \leq\left|\frac{A u\left(t_{1}\right)}{1+t_{1}}-\xi\right|+\left|\frac{A u\left(t_{2}\right)}{1+t_{2}}-\xi\right| \\
& <\varepsilon, \quad t_{1}, t_{2}>N .
\end{aligned}
$$

This implies that $A M$ is equiconvergent at $+\infty$.

Let $u_{n} \longrightarrow u$ as $n \longrightarrow+\infty$; then, there exists $r_{0}$ such that $\max _{n \in \mathbb{N} \backslash\{0\}}\left\{\left\|u_{n}\right\|,\|u\|\right\}<r_{0}, \mathbb{N}$ is a natural number set. By $\left(H_{2}\right)$, we have

$$
\begin{aligned}
& \left|\int_{0}^{\infty} G(\tau, \varsigma) a(\varsigma) f\left(\varsigma, u_{n}(\varsigma)\right) d \varsigma-\int_{0}^{\infty} G(\tau, \varsigma) a(\varsigma) f(\varsigma, u(\varsigma)) d \varsigma\right| \\
& \quad \leq \int_{0}^{\infty} G(\tau, \varsigma) a(\varsigma)\left|f\left(\varsigma, u_{n}(\varsigma)\right)-f(\varsigma, u(\varsigma))\right| d \varsigma \\
& \quad \leq 2 S_{f}^{r_{0}} \int_{0}^{\infty} G(\tau, \varsigma) a(\varsigma) d \varsigma<+\infty
\end{aligned}
$$

where

$$
S_{f}^{r_{0}}:=\sup \left\{f(t,(1+t) x):(t, x) \in[0,+\infty) \times\left[0, r_{0}\right]\right\}<+\infty .
$$

So, for any $\varepsilon>0$, we can find a sufficiently large $H_{0}>0$, such that

$$
S_{f}^{r_{0}} \int_{H_{0}}^{\infty} G(\tau, \varsigma) a(\varsigma) d \varsigma<\frac{\varepsilon}{4} .
$$

It follows from the Lebesgue dominated convergence theorem and continuity of $f$, we can get

$$
\begin{aligned}
& \left|\int_{0}^{H_{0}} G(\tau, \varsigma) a(\varsigma) f\left(\varsigma, u_{n}(\varsigma)\right) d \varsigma-\int_{0}^{H_{0}} G(\tau, \varsigma) a(\varsigma) f(\varsigma, u(\varsigma)) d \varsigma\right| \\
& \quad \leq \int_{0}^{H_{0}} G(\tau, \varsigma) a(\varsigma)\left|f\left(\varsigma, u_{n}(\varsigma)\right)-f(\varsigma, u(\varsigma))\right| d \varsigma \\
& \quad \longrightarrow 0, n \longrightarrow+\infty, \tau \in[0,+\infty) .
\end{aligned}
$$

So, for the above $\varepsilon>0$, there exists $N>0$, when $n>N_{0}$, we have

$$
\int_{0}^{H_{0}} G(\tau, \varsigma) a(\varsigma)\left|f\left(\varsigma, u_{n}(\varsigma)\right)-f(\varsigma, u(\varsigma))\right| d \varsigma<\frac{\varepsilon}{2} .
$$

Therefore, when $n>N_{0}$,

$$
\begin{aligned}
& \left|\int_{0}^{\infty} G(\tau, \varsigma) a(\varsigma) f\left(\varsigma, u_{n}(\varsigma)\right) d \varsigma-\int_{0}^{\infty} G(\tau, \varsigma) a(\varsigma) f(\varsigma, u(\varsigma)) d \varsigma\right| \\
& \quad \leq \int_{0}^{\infty} G(\tau, \varsigma) a(\varsigma)\left|f\left(\varsigma, u_{n}(\varsigma)-f \varsigma, u_{n}(\varsigma)\right)\right| d \varsigma \\
& \quad \leq \int_{0}^{H_{0}} G(\tau, \varsigma) a(\varsigma)\left|f\left(\varsigma, u_{n}(\varsigma)-f \varsigma, u_{n}(\varsigma)\right)\right| d \varsigma \\
& \quad+\int_{H_{0}}^{\infty} G(\tau, \varsigma) a(\varsigma)\left|f\left(\varsigma, u_{n}(\varsigma)-f \varsigma, u_{n}(\varsigma)\right)\right| d \varsigma \\
& \leq \frac{\varepsilon}{2}+2 S_{f}^{r_{0}} \int_{H_{0}}^{\infty} G(\tau, \varsigma) a(\varsigma) d \varsigma<\varepsilon,
\end{aligned}
$$

which implied that

$$
\begin{aligned}
& \mid \tau \varphi_{q}\left(\int_{0}^{\infty} G(\tau, \varsigma) a(\varsigma) f\left(\varsigma, u_{n}(\varsigma)\right) d \varsigma\right) \\
& \quad-\tau \varphi_{q}\left(\int_{0}^{\infty} G(\tau, \varsigma) a(\varsigma) f(\varsigma, u(\varsigma)) d \varsigma\right) \mid \longrightarrow 0
\end{aligned}
$$

as $n \longrightarrow+\infty, \tau \in[0,+\infty)$. Since

$$
\begin{aligned}
& \mid \int_{0}^{t} \int_{s}^{\infty} \varphi_{q}\left(\int_{0}^{\infty} G(\tau, \varsigma) a(\varsigma) f\left(\varsigma, u_{n}(\varsigma)\right) d \varsigma\right) d \tau d s \\
& \quad-\int_{0}^{t} \int_{s}^{\infty} \varphi_{q}\left(\int_{0}^{\infty} G(\tau, \varsigma) a(\varsigma) f(\varsigma, u(\varsigma)) d \varsigma\right) d \tau d s \mid \\
& \leq 2 \varphi_{q}\left(s_{f}^{r_{0}}\right) \int_{0}^{\infty} s \varphi_{q}\left(\int_{0}^{\infty} G(s, \varsigma) a(\varsigma) d \varsigma\right) d s<+\infty .
\end{aligned}
$$
we get

Then, by the Lebesgue dominated convergence theorem,

$$
\begin{aligned}
\left\|A u_{n}-A u\right\| & \\
= & \sup _{t \longrightarrow+\infty} \frac{1}{1+t} \mid \frac{1}{1-\int_{0}^{\infty} g(t) d t} \int_{0}^{\infty} g(t) \\
& \quad \int_{0}^{t} \int_{s}^{\infty}\left(\varphi_{q}\left(\int_{0}^{\infty} G(\tau, \varsigma) a(\varsigma) f\left(\varsigma, u_{n}(\varsigma)\right) d \varsigma\right)\right. \\
& \left.-\varphi_{q}\left(\int_{0}^{\infty} G(\tau, \varsigma) a(\varsigma) f(\varsigma, u(\varsigma)) d \varsigma\right)\right) d \tau d s d t \\
& +\int_{0}^{t} \int_{s}^{\infty}\left(\varphi_{q}\left(\int_{0}^{\infty} G(\tau, \varsigma) a(\varsigma) f\left(\varsigma, u_{n}(\varsigma)\right) d \varsigma\right)\right. \\
& \left.-\varphi_{q}\left(\int_{0}^{\infty} G(\tau, \varsigma) a(\varsigma) f(\varsigma, u(\varsigma)) d \varsigma\right)\right) d \tau d s \mid
\end{aligned}
$$




$$
\begin{aligned}
\leq & \frac{1}{1-\int_{0}^{\infty} g(t) d t} \int_{0}^{\infty} g(t) \int_{0}^{t} \int_{s}^{\infty} \\
& \cdot \mid \varphi_{q}\left(\int_{0}^{\infty} G(\tau, \varsigma) a(\varsigma) f\left(\varsigma, u_{n}(\varsigma)\right) d \varsigma\right) \\
& -\varphi_{q}\left(\int_{0}^{\infty} G(\tau, \varsigma) a(\varsigma) f(\varsigma, u(\varsigma)) d \varsigma\right) \mid d \tau d s d t \\
& +\int_{0}^{t} \int_{s}^{\infty} \mid \varphi_{q}\left(\int_{0}^{\infty} G(\tau, \varsigma) a(\varsigma) f\left(\varsigma, u_{n}(\varsigma)\right) d \varsigma\right) \\
& -\varphi_{q}\left(\int_{0}^{\infty} G(\tau, \varsigma) a(\varsigma) f(\varsigma, u(\varsigma)) d \varsigma\right) \mid d \tau d s \\
\leq & \left.\quad \frac{\int_{0}^{\infty} g(t) d t}{1-\int_{0}^{\infty} g(t) d t}+1\right) \int_{0}^{\infty} s \\
& \cdot \mid \varphi_{q}\left(\int_{0}^{\infty} G(s, \varsigma) a(\varsigma) f\left(\varsigma, u_{n}(\varsigma)\right) d \varsigma\right) \\
& -\varphi_{q}\left(\int_{0}^{\infty} G(s, \varsigma) a(\varsigma) f(\varsigma, u(\varsigma)) d \varsigma\right) \mid d s \\
\longrightarrow & 0, n \longrightarrow+\infty .
\end{aligned}
$$

Therefore, $A: K \longrightarrow K$ is continuous. In conclusion, by Lemma 4 , we know that $A: K \longrightarrow K$ is completely continuous. The proof is completed.

Theorem 6. Assume that $\left(\mathrm{H}_{0}\right)-\left(\mathrm{H}_{2}\right)$ hold and there exists $d>2 m$ which satisfies the following condition:

$\left(H_{3}\right) f\left(t, x_{1}\right) \leq f\left(t, x_{2}\right), t \in[0,+\infty), 0 \leq x_{1} \leq x_{2}$.

$\left(H_{4}\right) f(t,(1+t) x) \leq \varphi_{p}(d / 2 l),(t, x) \in[0,+\infty) \times[0, d]$,

where

$$
\begin{aligned}
& m=\xi\left(1+\frac{\int_{0}^{\infty} \operatorname{tg}(t) d t}{1-\int_{0}^{\infty} g(t) d t}\right) \\
& l=\frac{1}{1-\int_{0}^{\infty} g(t) d t} \int_{0}^{\infty} s \varphi_{q}\left(\int_{0}^{\infty} G(s, \varsigma) a(\varsigma) d \varsigma\right) d s
\end{aligned}
$$

Then, BVP (2) has the maximal and minimal positive solutions $\mu^{*}$ and $\bar{\mu}^{*}$ on $[0,+\infty)$, such that

$$
\begin{aligned}
& 0<\sup _{t \in[0,+\infty)} \frac{\left|\mu^{*}(t)\right|}{1+t} \leq d, \\
& 0<\sup _{t \in[0,+\infty)} \frac{\left|\bar{\mu}^{*}(t)\right|}{1+t} \leq d .
\end{aligned}
$$

Moreover, for initial values

$$
\begin{aligned}
\mu_{0}(t) & =\frac{d}{2}+\xi\left(t+\frac{\int_{0}^{\infty} \operatorname{tg}(t) d t}{1-\int_{0}^{\infty} g(t) d t}\right), \\
\bar{\mu}_{0}(t) & =0, \\
t & \in[0,+\infty),
\end{aligned}
$$

define the iterative sequences $\left\{\mu_{n}\right\}$ and $\left\{\bar{\mu}_{n}\right\}$ by

$$
\begin{aligned}
\mu_{n}= & \frac{1}{1-\int_{0}^{\infty} g(t) d t}\left(\xi \int_{0}^{\infty} \operatorname{tg}(t) d t+\int_{0}^{\infty} g(t)\right. \\
& \left.\cdot \int_{0}^{t} \int_{s}^{\infty} \varphi_{q}\left(\int_{0}^{\infty} G(\tau, \varsigma) a(\varsigma) f\left(\varsigma, \mu_{n-1}(\varsigma)\right) d \varsigma\right) d \tau d s d t\right) \\
& +\int_{0}^{t} \int_{s}^{\infty} \varphi_{q}\left(\int_{0}^{\infty} G(\tau, \varsigma) a(\varsigma) f\left(\varsigma, \mu_{n-1}(\varsigma)\right) d \varsigma\right) d \tau d s+\xi t \\
\bar{\mu}_{n}= & \frac{1}{1-\int_{0}^{\infty} g(t) d t}\left(\xi \int_{0}^{\infty} t g(t) d t+\int_{0}^{\infty} g(t)\right. \\
& \left.\cdot \int_{0}^{t} \int_{s}^{\infty} \varphi_{q}\left(\int_{0}^{\infty} G(\tau, \varsigma) a(\varsigma) f\left(\varsigma, \bar{\mu}_{n-1}(\varsigma)\right) d \varsigma\right) d \tau d s d t\right) \\
& +\int_{0}^{t} \int_{s}^{\infty} \varphi_{q}\left(\int_{0}^{\infty} G(\tau, \varsigma) a(\varsigma) f\left(\varsigma, \bar{\mu}_{n-1}(\varsigma)\right) d \varsigma\right) d \tau d s+\xi t .
\end{aligned}
$$

Then,

$$
\begin{aligned}
& \lim _{n \rightarrow+\infty} \sup _{t \in[0,+\infty)} \frac{\left|\mu_{n}(t)-\mu^{*}(t)\right|}{1+t}=0, \\
& \lim _{n \longrightarrow+\infty} \sup _{t \in[0,+\infty)} \frac{\left|\bar{\mu}_{n}(t)-\bar{\mu}^{*}(t)\right|}{1+t}=0 .
\end{aligned}
$$

Proof. From Lemma 5, we know that $A: K \longrightarrow K$ is completely continuous. For any $u_{1}, u_{2} \in K$ with $u_{1} \leq u_{2}$, from the definition of $A$ and $\left(H_{3}\right)$, we know that $A u_{1} \leq A u_{2}$. Let $K_{d}=\{u \in K:\|u\| \leq d\}$. In what follows, we firstly prove $A$ $: K_{d} \longrightarrow K_{d}$. In fact, for any $u \in K_{d}$, we have $0 \leq u(t) /(1+t)$ $\leq d, t \in[0,+\infty)$. By $\left(H_{4}\right)$, we know that $f(t, x) \leq \varphi_{p}(d / 2 l)$, $(t, x) \in[0,+\infty) \times[0, d]$. Also, by $\left(H_{4}\right)$, we have

$$
\mu_{n+1}(t) \leq \mu_{n}(t), \quad t \in[0,+\infty), n=0,1,2, \cdots
$$

Thus, we have proved that $A: K_{d} \longrightarrow K_{d}$. Let $\mu_{0}(t)$ $=(d / 2)+\xi\left(t+\left(\left(\int_{0}^{\infty} \operatorname{tg}(t) d t\right) /\left(1-\int_{0}^{\infty} g(t) d t\right)\right)\right), t \in[0,+\infty)$, and then, $\mu_{0}(t) \in K_{d}$. Let $\mu_{1}=A \mu_{0}, \mu_{2}=A \mu_{1}=A^{2} \mu_{0}$; by Theorem 6, we have $\mu_{1}, \mu_{2} \in K_{d}$. Denote $\mu_{n+1}=A \mu_{n}=A^{n}$ $\mu_{0}, n=1,2, \cdots$. Since $A: K_{d} \longrightarrow K_{d}$, we have $\mu_{n} \in A\left(K_{d}\right)$ $\subset K_{d}$. It follows from the complete continuity of $A$ that $\left\{\mu_{n}\right\}_{n=1}^{\infty}$ is a sequentially compact set. By $\left(H_{4}\right)$, we have

$$
\begin{aligned}
\mu_{1}(t)= & A \mu_{0}(t)=\frac{1}{1-\int_{0}^{\infty} g(t) d t}\left(\xi \int_{0}^{\infty} t g(t) d t+\int_{0}^{\infty} g(t)\right. \\
& \left.\cdot \int_{0}^{t} \int_{s}^{\infty} \varphi_{q}\left(\int_{0}^{\infty} G(\tau, \varsigma) a(\varsigma) f\left(\varsigma, \mu_{0}(\varsigma)\right) d \varsigma\right) d \tau d s d t\right) \\
& +\int_{0}^{t} \int_{s}^{\infty} \varphi_{q}\left(\int_{0}^{\infty} G(\tau, \varsigma) a(\varsigma) f\left(\varsigma, \mu_{0}(\varsigma)\right) d d \varsigma\right) d \tau d s+\xi t
\end{aligned}
$$




$$
\begin{aligned}
\leq & \frac{1}{1-\int_{0}^{\infty} g(t) d t} \int_{0}^{\infty} g(t) d t \int_{0}^{\infty} s \varphi_{q} \\
& \cdot\left(\int_{0}^{\infty} G(s, \varsigma) a(\varsigma) f\left(\varsigma, \mu_{0}(\varsigma)\right) d \varsigma\right) d s \\
& +\int_{0}^{\infty} s \varphi_{q}\left(\int_{0}^{\infty} G(s, \varsigma) a(\varsigma) f\left(\varsigma, \mu_{0}(\varsigma)\right) d d \varsigma\right) d s \\
& +\xi\left(\frac{\int_{0}^{\infty} t g(t) d t}{1-\int_{0}^{\infty} g(t) d t}\right)+\xi t \leq \frac{d}{2 l\left(1-\int_{0}^{\infty} g(t) d t\right)} \\
& \cdot \int_{0}^{\infty} s \varphi_{q}\left(\int_{0}^{\infty} G(s, \varsigma) a(\varsigma) d \varsigma\right) d s \\
& +\xi\left(t+\frac{\int_{0}^{\infty} t g(t) d t}{1-\int_{0}^{\infty} g(t) d t}\right) \\
\leq & \frac{d}{2}+\xi\left(t+\frac{\int_{0}^{\infty} t g(t) d t}{1-\int_{0}^{\infty} g(t) d t}\right)=\mu_{0}(t) .
\end{aligned}
$$

Together with $\left(H_{4}\right)$, we also have $\mu_{2}(t)=A \mu_{1}(t) \leq A$ $\mu_{0}(t)=\mu_{1}(t), t \in[0,+\infty)$. By induction, we obtain

$$
\mu_{n+1}(t) \leq \mu_{n}(t), \quad t \in[0,+\infty), n=0,1,2, \cdots .
$$

Therefore, there exists $\mu^{*} \in K_{d}$ such that $\mu_{n} \longrightarrow \mu^{*}$ as $n \longrightarrow+\infty$. Applying the continuity of $A$ and $\mu_{n+1}=A \mu_{n}$, we get that $A \mu^{*}=\mu^{*}$.

On the other hand, let $\bar{\mu}_{0}(t)=0, t \in[0,+\infty)$, and then $\bar{\mu}_{0}(t) \in K_{d}$. Let $\bar{\mu}_{1}=A \bar{\mu}_{0}, \bar{\mu}_{2}=A \bar{\mu}_{1}=A^{2} \bar{\mu}_{0}$; then, by Theorem 6 , we have $\bar{\mu}_{1}, \bar{\mu}_{2} \in K_{d}$. Denote $\bar{\mu}_{n+1}=A \bar{\mu}_{n}=A^{n} \bar{\mu}_{0},=1,2, \cdots$. Since $A: K_{d} \longrightarrow K_{d}$, we have $\bar{\mu}_{n} \in A\left(K_{d}\right) \subset K_{d}$. It follows from the complete continuity of $A$ that $\left\{\bar{\mu}_{n}\right\}_{n=1}^{\infty}$ is a sequentially compact set. Since $\bar{\mu}_{1}=A \bar{\mu}_{0} \in K_{d}$, we have

$$
\bar{\mu}_{2}(t)=A \bar{\mu}_{1}(t)=A 0(t) \geq 0, t \in[0,+\infty) .
$$

By induction, we get

$$
\bar{\mu}_{n+1}(t) \geq \bar{\mu}_{n}(t), \in[0,+\infty), \quad n=1,2, \cdots .
$$

Thus, there exists $\bar{\mu}^{*} \in K$ such that $\bar{\mu}_{n} \longrightarrow \bar{\mu}^{*}$ as $n \longrightarrow+$ $\infty$. Applying the continuity of $A$ and $\bar{\mu}_{n+1}=A \bar{\mu}_{n}$, we get that $A \bar{\mu}^{*}=\bar{\mu}^{*}$.

Now, we are in a position to show that $\mu^{*}$ and $\bar{\mu}^{*}$ are the maximal and minimal positive solutions of BVP (2) in $\left(0,(d / 2)+\xi\left(t+\left(\left(\int_{0}^{\infty} t g(t) d t\right) /\left(1-\int_{0}^{\infty} g(t) d t\right)\right)\right)\right]$. Let $u \in[0$, $\left.(d / 2)+\xi\left(t+\left(\left(\int_{0}^{\infty} t g(t) d t\right) /\left(1-\int_{0}^{\infty} g(t) d t\right)\right)\right)\right]$ be any solution of BVP (2), that is, $A u=u$. Noting that $A$ is nondecreasing and $\bar{\mu}_{0}(t)=0 \leq u(t) \leq(d / 2)+\xi\left(t+\left(\left(\int_{0}^{\infty} t g(t) d t\right) /\right.\right.$ $\left.\left.\left(1-\int_{0}^{\infty} g(t) d t\right)\right)\right)=\mu_{0}(t)$, then we have $\bar{\mu}_{1}(t)=A \bar{\mu}_{0}(t) \leq u$ $(t) \leq A \mu_{0}(t)=\mu_{1}(t)$, for all $t \in[0,+\infty)$. By induction, we have

$$
\bar{\mu}_{n}(t) \leq u(t) \leq \mu_{n}(t), \quad n=1,2,3, \cdots
$$

Since $\mu^{*}=\lim _{n \longrightarrow+\infty} \mu_{n}, \bar{\mu}^{*}=\lim _{n \longrightarrow+\infty} \bar{\mu}_{n}$, it follows from (42)-(45) that

$$
\bar{\mu}_{0} \leq \bar{\mu}_{1} \leq \cdots \bar{\mu}_{n} \leq \cdots \leq \bar{\mu}^{*} \leq u \leq \mu^{*} \leq \cdots \leq \mu_{n} \leq \cdots \leq \mu_{1} \leq \mu_{0} .
$$

In virtue of $f(t, 0), t \in[0,+\infty)$, then the zero function is not the solution of BVP (2). Therefore, by (46), we know that $\mu^{*}$ and $\bar{\mu}^{*}$ are the maximal and minimal positive solutions of BVP (2) in $\left(0,(d / 2)+\xi\left(t+\left(\left(\int_{0}^{\infty} t g(t) d t\right) /\left(1-\int_{0}^{\infty}\right.\right.\right.\right.$ $g(t) d t)))$ ], which can be obtained by the corresponding iterative sequences $\mu_{n}=A \mu_{n-1}, \bar{\mu}_{n}=A \bar{\mu}_{n-1}$. The proof is completed.

Remark 1. The iterative schemes in Theorem 6 start with a known simple linear function and the zero function, respectively. This is very convenient in application. So Theorem 6 is very interesting and importance. Similarly, we can obtain Theorem 7 .

Theorem 7. Assume that $\left(H_{0}\right)-\left(H_{3}\right)$ hold and there exist $d_{n}>d_{n-1}>\cdots>d_{2}>d_{1}>2 m$ satisfying the following condition:

$\left(H_{4}^{\prime}\right) f(t,(1+t) x) \leq \varphi_{p}\left(d_{j} / 2 l\right),(t, x) \in[0,+\infty) \times\left[0, d_{j}\right], j$ $=1,2, \cdots, n . m, l$ are defined as Theorem 6. Then, BVP (2) has the maximal and minimal positive solutions $\mu_{j}^{*}$ and $\bar{\mu}_{j}^{*}$, on $[0,+\infty)$, such that

$$
\begin{aligned}
& 0<\sup _{t \in[0,+\infty)} \frac{\left|\mu_{j}^{*}(t)\right|}{1+t} \leq d, \\
& 0<\sup _{t \in[0,+\infty)} \frac{\left|\bar{\mu}_{j}^{*}(t)\right|}{1+t} \leq d .
\end{aligned}
$$

\section{Moreover, for initial values}

$$
\begin{gathered}
\mu_{j 0}(t)=\frac{d_{j}}{2}+\xi\left(t+\frac{\int_{0}^{\infty} t g(t) d t}{1-\int_{0}^{\infty} g(t) d t}\right) \\
\bar{\mu}_{j 0}(t)=0 \\
t \in[0,+\infty)
\end{gathered}
$$

define the iterative sequences $\left\{\mu_{j n}\right\}$ and $\left\{\bar{\mu}_{\text {jn }}\right\}$ by

$$
\begin{aligned}
\mu_{j n}= & \frac{1}{1-\int_{0}^{\infty} g(t) d t}\left(\xi \int_{0}^{\infty} t g(t) d t+\int_{0}^{\infty} g(t)\right. \\
& \left.\cdot \int_{0}^{t} \int_{s}^{\infty} \varphi_{q}\left(\int_{0}^{\infty} G(\tau, \varsigma) a(\varsigma) f\left(\varsigma, \mu_{j(n-1)}(\varsigma)\right) d \varsigma\right) d \tau d s d t\right) \\
& +\int_{0}^{t} \int_{s}^{\infty} \varphi_{q}\left(\int_{0}^{\infty} G(\tau, \varsigma) a(\varsigma) f\left(\varsigma, \mu_{j(n-1)}(\varsigma)\right) d \varsigma\right) \\
& \cdot d \tau d s+\xi t
\end{aligned}
$$




$$
\begin{aligned}
\bar{\mu}_{j n}= & \frac{1}{1-\int_{0}^{\infty} g(t) d t}\left(\xi \int_{0}^{\infty} t g(t) d t+\int_{0}^{\infty} g(t)\right. \\
& \left.\cdot \int_{0}^{t} \int_{s}^{\infty} \varphi_{q}\left(\int_{0}^{\infty} G(\tau, \varsigma) a(\varsigma) f\left(\varsigma, \mu_{j(n-1)}(\varsigma)\right) d \varsigma\right) d \tau d s d t\right) \\
& +\int_{0}^{t} \int_{s}^{\infty} \varphi_{q}\left(\int_{0}^{\infty} G(\tau, \varsigma) a(\varsigma) f\left(\varsigma, \mu_{j(n-1)}(\varsigma)\right) d \varsigma\right) \\
& \cdot d \tau d s+\xi t
\end{aligned}
$$

Then,

$$
\begin{aligned}
& \lim _{n \longrightarrow+\infty} \sup _{t \in[0,+\infty)} \frac{\left|\mu_{j n}(t)-\mu_{j}^{*}(t)\right|}{1+t}=0, \\
& \lim _{n \longrightarrow+\infty} \sup _{t \in[0,+\infty)} \frac{\left|\bar{\mu}_{j n}(t)-\bar{\mu}_{j}^{*}(t)\right|}{1+t}=0 .
\end{aligned}
$$

Remark 2. We note that $\mu^{*}$ and $\bar{\mu}^{*}$ in Theorem 6 may coincide; then, BVP (2) has only one solution in $K_{d}$. Similarly, positive solutions $\mu_{j}^{*}$ and $\bar{\mu}_{j}^{*}$ in Theorem 7 may also coincide.

\section{Example}

Consider the following BVP

$$
\left\{\begin{array}{l}
\left(\left|u^{\prime \prime}(t)\right|^{-1 / 2} u^{\prime \prime}(t)\right)^{\prime \prime}-\left|u^{\prime \prime}(t)\right|^{-1 / 2} u^{\prime \prime}(t)=a(t) f(t, u(t)), t \in(0,+\infty), \\
u(0)=\int_{0}^{\infty} \frac{1}{(1+t)^{3}} u(t) d t, \lim _{t \rightarrow+\infty} u^{\prime}(t)=\frac{\sqrt{3}}{4},,^{\prime \prime}(0)=\lim _{t \longrightarrow+\infty} u^{\prime \prime}(t)=0 .
\end{array}\right.
$$

Obviously, we know that $p=3 / 2, k=1, \xi=\sqrt{3} / 4, g(t)=$ $1 /(1+t)^{3}$, so we can get

$$
\begin{aligned}
\int_{0}^{\infty} g(t) d t & =\int_{0}^{\infty} \frac{1}{(1+t)^{3}} d t=\frac{1}{2}<1, \int_{0}^{\infty} t g(t) d t \\
& =\int_{0}^{\infty} \frac{t}{(1+t)^{3}} d t=\frac{1}{2}<+\infty, \\
G(t, s) & =\frac{1}{2}\left\{\begin{array}{l}
e^{-s}\left(e^{t}-e^{-t}\right), 0 \leq t \leq s<+\infty, \\
e^{-t}\left(e^{s}-e^{-s}\right), 0 \leq s \leq t<+\infty .
\end{array}\right.
\end{aligned}
$$

Let

$$
\begin{aligned}
a(t) & =e^{-2 t}, f(t, x) \\
& = \begin{cases}|\sin (55 t+21)|+\frac{1}{144}\left(\frac{x}{1+t}\right)^{3}, & x \leq 2, \\
|\sin (55 t+21)|+\frac{1}{144}\left(\frac{2}{1+t}\right)^{3}, & x \geq 2 .\end{cases}
\end{aligned}
$$

By computation, we get

$$
\begin{aligned}
& \int_{0}^{\infty} G(s, s) a(s) d s \leq \frac{1}{2} \int_{0}^{\infty} e^{-2 s} d s=\frac{1}{4}<+\infty, \\
& \int_{0}^{\infty} s \varphi_{q}\left(\int_{0}^{\infty} G(s, \varsigma) a(\varsigma) d \varsigma\right) d s \\
& =\int_{0}^{\infty} s \varphi_{q}\left(\int_{0}^{s} G(s, \varsigma) a(\varsigma) d \varsigma\right. \\
& \left.\quad+\int_{s}^{\infty} G(s, \varsigma) a(\varsigma) d \varsigma\right) d s=\frac{13}{432}<+\infty .
\end{aligned}
$$

So, the conditions $\left(H_{0}\right)-\left(H_{3}\right)$ hold. For $m=\sqrt{3} / 2, l=$ $13 / 216$. Take $d=10$, it follows that

$$
\begin{aligned}
f_{1}(t,(1+t) x) & \leq \frac{10}{9}<\varphi_{p}\left(\frac{d}{2 l}\right)=\varphi_{p}\left(\frac{10}{2 \cdot(13 / 216)}\right) \\
& =\sqrt{\frac{1080}{13}}, \quad(t, x) \in[0,+\infty) \times[0,10] .
\end{aligned}
$$

Hence, condition $\left(\mathrm{H}_{4}\right)$ holds, that is, all conditions of Theorem 6 are satisfied. Therefore, BVP (51) has the minimal and maximal positive solutions in $(0,5+(\sqrt{3} / 4)(t+1)]$, which can be obtained by two explicit monotone iterative sequences.

\section{Data Availability}

No data was generated or used during the study; this paper was not about data.

\section{Conflicts of Interest}

The authors declare that they have no conflicts of interest.

\section{Acknowledgments}

The author was supported financially by the National Natural Science Foundation of China (11701252), NSF of Shandong Province (ZR2019YQ05), and the Science Research Foundation for Doctoral Authorities of Linyi University (LYDX2016BS080).

\section{References}

[1] J. I. Diaz, "Nonlinear partial differential equations and free boundaries," in Vol. 1: Elliptic Equations, in: Research Notes in Mathematics, vol. 106, Pitman Advanced Publishing Program, Boston, 1985.

[2] D. O’Regan, B. Yan, and R. P. Agarwal, "Solutions in weighted spaces of singular boundary value problems on the half- line," Journal of Computational and Applied Mathematics, vol. 205, no. 2, pp. 751-763, 2007.

[3] X. Zhao and W. Ge, "Existence of at least three positive solutions formulti-point boundary value problem on infinite intervals with $p$-Laplacian operator," Journal of Applied Mathematics and Computing, vol. 28, no. 1-2, pp. 391-403, 2008. 
[4] K. Chua, D. Haib, and R. Shivaji, "Uniqueness for a class of $p$ -Laplacian problems when the reaction term tends to zero at infinity," Journal of Mathematical Analysis and Applications, vol. 494, no. 2, article 124576, 2021.

[5] J. Sun and H. Chen, "Multiple positive solutions for multipoint boundary value problems with a $p$-Laplacian on the half-line," Journal of Applied Mathematics and Computing, vol. 33, no. 1-2, pp. 173-191, 2010.

[6] H. Lian, P. J. Y. Wong, and S. Yang, "Solvability of three-point boundary value problems at resonance with a -Laplacian on finite and infinite intervals," Abstract and Applied Analysis, vol. 2012, Article ID 658010, 16 pages, 2012.

[7] J. Jiang, L. Liu, and Y. Wu, "Positive solutions for $p$-Laplacian fourth-order differential system with integral boundary conditions," Discrete Dynamics in Nature and Society, vol. 2012, Article ID 293734, 19 pages, 2012.

[8] J. Li, B. Liu, and L. Liu, "Solutions for a boundary value problem at resonance on $[0, \infty)$," Mathematical and Computer Modelling, vol. 58, no. 11-12, pp. 1769-1776, 2013.

[9] W. Jiang, "Solvability for $p$-Laplacian boundary value problem at resonance on the half-line," Boundary Value Problems, vol. 2013, no. 1, Article ID 207, 2013.

[10] L. Wang, T. Han, and J. Wang, "Infinitely many solutions for Schrödinger-Choquard-Kirchhoff equations involving the fractional p-Laplacian," Acta Mathematica Sinica, English Series, vol. 37, no. 2, pp. 315-332, 2021.

[11] X. Hao, P. Zhang, and L. Liu, "Iterative solutions of singular $(k, n-k)$ conjugate boundary value problems with dependence on the derivatives," Applied Mathematics Letters, vol. 27, no. 8, pp. 64-69, 2014.

[12] L. Liu, X. Zhang, L. Liu, and Y. Wu, "Iterative positive solutions for singular nonlinear fractional differential equation with integral boundary conditions," Advances in Difference Equations, vol. 2016, no. 1, Article ID 154, 2016.

[13] L. Ma, "On the Poisson equation of $p$-Laplacian and the nonlinear Hardy-type problems," Zeitschrift für angewandte Mathematik und Physik, vol. 72, no. 1, p. 34, 2021.

[14] X. Hao, H. Wang, L. Liu, and Y. Cui, "Positive solutions for a system of nonlinear fractional nonlocal boundary value problems with parameters and $p$-Laplacian operator," Boundary Value Problems, vol. 2017, no. 1, Article ID 182, 2017.

[15] J. Ren and C. Zhai, "Solvability for $p$-Laplacian generalized fractional coupled systems with two-sided memory effects," Mathematical Methods in the Applied Sciences, vol. 43, no. 15, pp. 8797-8822, 2020.

[16] C. Zhai, Y. Ma, and H. Li, "Unique positive solution for a $p$ -Laplacian fractional differential boundary value problem involving Riemann-Stieltjes integral," AIMS Mathematics, vol. 5, no. 5, pp. 4754-4769, 2020.

[17] C. Yang and X. Zhu, "Positive solutions depending on parameters for a nonlinear fractional system with -Laplacian operators," Advances in Mathematical Physics, vol. 2020, Article ID 9563791, 8 pages, 2020.

[18] S. Liang and J. Zhang, "The existence of countably many positive solutions for one-dimensional $p$-Laplacian with infinitely many singularities on the half-line," Applied Mathematics and Computation, vol. 201, no. 1-2, pp. 210-220, 2008.

[19] J. Xu and Z. Yang, "Positive solutions for a fourth order $p$ -Laplacian boundary value problem," Nonlinear Analysis, vol. 74, no. 7, pp. 2612-2623, 2011.
[20] M. Zima, "On positive solutions of boundary value problems on the half-line," Journal of Mathematical Analysis and Applications, vol. 259, no. 1, pp. 127-136, 2001.

[21] Y. Liu, "Boundary value problem for second order differential equations on unbounded domain," Acta Analysis Functionalis Applicata, vol. 4, no. 3, pp. 211-216, 2002.

[22] C. Corduneanu, Integral Equations and Stability of Feedback Systems, Academic Press, New York, NY, USA, 1973. 